\title{
El Grupo Visegrado: una visión de Europa Central. Conversación con Lech Walesa ${ }^{1}$
}

\author{
The Visegrad Group: a vision of Central Europe. Conversation with Lech Walesa
}

Alejandro, Negrín²; Pablo, Lozano ${ }^{3}$

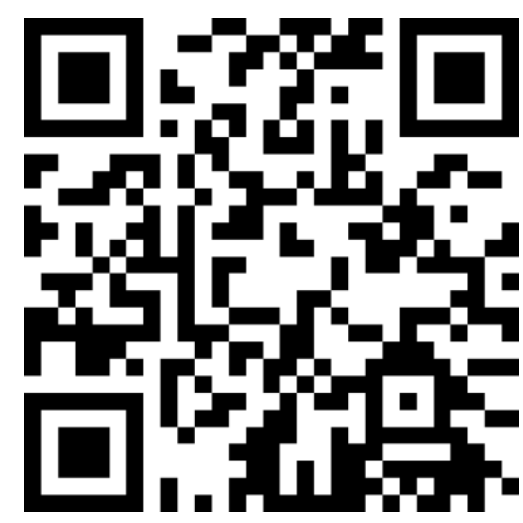

.................................

Fecha de recibido: 08-04-2021

Fecha de aceptado: 21-06-2021

\section{(c) BY-NC-ND}

Esta obra está bajo una licencia de Creative Commons Reconocimiento-NoComercialSinObraDerivada 4.0 Internacional

\begin{abstract}
RESUMEN
Este trabajo busca presentar, a partir de un testimonio directo, una visión legible y actualizada del concepto de Europa Central, como subregión diferenciada en el contexto de la Unión Europea. Entender la construcción de una nueva Europa Central luego del fin de la era comunista -caracterizada hoy por democracias liberales y economías de mercado consolidadas y con gran potencial- es uno de los retos más significativos de la política exterior de México. El concepto instrumental que orienta este trabajo es la construcción y funcionamiento del Grupo Visegrado, formado en 1991 e integrado por Polonia, República Checa, Eslovaquia y Hungría como la expresión política y de cooperación más consistente y clara de Europa Central en la actualidad. La reflexión que presenta este trabajo tiene como punto de partida un diálogo directo de los autores con uno de los artífices del Grupo Visegrado y actor central del fin del comunismo en Europa: el célebre líder obrero y expresidente polaco Lech Walesa. A treinta años de distancia de la creación del Grupo Visegrado, resulta fundamental conocer su perspectiva.
\end{abstract}

Palabras claves: Europa Central, Grupo Visegrado, Lech Walesa, Vaclav Havel, Política exterior de México.

\begin{abstract}
This work seeks to present, based on a direct testimony, a readable and updated vision of the concept of Central Europe, as a differentiated subregion in the context of the European Union.

To understand the construction of a new Central Europe after the end of the communist era - characterized today by liberal democracies and consolidated market economies with great potential - is one of the most significant challenges of Mexico's foreign policy. The instrumental concept that guides this work is the construction and functioning of the Visegrad Group, formed in 1991 and constituted by Poland, the Czech Republic, Slovakia and Hungary as the clearer and most consistent political and cooperation expression in Central Europe today. The reflection presented in this work has as its starting point a direct dialogue between the authors with one of the architects of the Visegrad Group and a central actor in the end of communism in Europe: the famous worker leader and former Polish president Lech Walesa. Thirty years after the creation of the Visegrad Group, it is essential to know his perspective.
\end{abstract}

Keywords: Central Europe, Visegrad Group, Lech Walesa, Vaclav Havel, Foreign Policy of Mexico.

Cómo referenciar este artículo:

Negrín., A. \& Lozano., P. (2022). El Grupo Visegrado: una visión de Europa Central. Conversación con Lech Walesa. Revista Política, Globalidad y Ciudadanía, 8(15), 130-151. https://doi.org/10.29105/pgc8.15-7

\footnotetext{
${ }^{1}$ Este artículo es producto del proyecto: "El Grupo Visegrado: una visión de Europa Central. Conversación con Lech Walesa".

${ }^{2}$ Embajada de México en Polonia, Polonia. Maestro en Relaciones Internacionales por el Instituto Universitario Ortega y Gasset de Madrid. Embajador de México en Polonia, Email: anegrin@sre.gob.mx. Orcid: 0000-0002-8583-6514.

${ }^{3}$ Embajada de México en Polonia, Polonia. Maestro en Negocios Europeos por la Ecole des Hautes Etudes Commerciales du Nord. Jefe de Cancillería en la Embajada de México en Polonia, Email: plozano@sre.gob.mx. Orcid: 0000-0003-4026-8557.
}

130 Revista Política, Globalidad y Ciudadanía | Vol. 8, Núm. 15, enero - junio 2022 | ISSN 2395-8448 | http://revpoliticas.uanl.mx/ 


\section{1.- INTRODUCCIÓN}

A pesar de la importancia y peso específico de la Unión Europea en el contexto internacional (con sólo el $6.9 \%$ de la población mundial, su comercio con el resto del planeta representa aproximadamente el $15.6 \%$ de las importaciones y exportaciones globales y cerca del $15.5 \%$ del producto interno bruto mundial), algunas de sus regiones -o subregiones- son prácticamente desconocidas. Ello aplica para diversas naciones, pero también para México. Una de las regiones que representan un enorme potencial en todos los ámbitos es la de Europa Central.

Europa Central es una subregión poco entendida por diversas razones. Una de carácter histórico, muy significativa, es que se trata de una de las regiones en el mundo que ha registrado más modificaciones a sus fronteras a lo largo de la historia y, en particular, en la historia contemporánea. Mucho más que Europa occidental. Lee y Bideleux señalan que "conceptualizar y construir geografías europeas, físicas y simbólicas, es una preocupación constante”. Si se piensa, por ejemplo, en la geopolítica que dejó la Segunda Guerra Mundial, es claro que el rol de la Unión Soviética como factor para la derrota de la Alemania nazi, "condujo a reconfiguraciones sustanciales de las fronteras y composiciones étnicas de los estados europeos", incluyendo la incorporación al bloque soviético de la mayoría de los territorios y naciones que pertenecieron en el periodo de las entreguerras a Polonia, Checoslovaquia, Hungría y Rumania así como la anexión a las Repúblicas Soviéticas Socialistas de Ucrania y Belarús e, incluso, transferencia de la región de Vilnus a la República Soviética Socialista de Lituania, entre tantas modificaciones limítrofes en la zona.

En 1954 el longevo geógrafo Karl A. Sinnhuber (Sinnhuber, K. 1954) decía que Europa Central era una región muy difícil de entender desde fuera: “...en vista de los grandes cambios en las fronteras políticas y el panorama cultural de Europa que han tenido lugar durante el pasado reciente, es posible que debamos modificar nuestras ideas en cuanto a la extensión de Europa Central. Pero sólo podemos tomar una decisión cuando hayamos reexaminado las formas en que el término se ha utilizado anteriormente".

Sinnhuber recordaba también a autores escépticos de la existencia misma de esa subregión europea. Decía que “el erudito austriaco Erwin Hanslik afirmó enfáticamente durante la Primera Guerra Mundial que Europa Central era sólo un fantasma de la imaginación y que el Este comenzó sin ninguna 
transición a lo largo de una línea desde Trieste pasando por Viena, Praga, Breslau (nota de los autores: actual Wroclaw, Polonia) hasta Königsberg”.

Lee y Bideleux señalan, por su parte, que "Las nociones de Europa se han manifestado como camaleónicas en muchas formas y disfraces", ya que "no hay una definición clara e indiscutible de sus límites” (Lee, C. \& Bideleux, R. 2012).

El desconocimiento sobre la región de Europa Central se extiende a la historia más reciente y, en particular, a la era que se abrió con el fin del comunismo y la caída del muro de Berlín, así como el fin de la Guerra Fría y del llamado Pacto de Varsovia, fenómenos, cada uno de éstos, de gran profundidad y extensión.

Es cierto que, en los primeros años de su vida postsoviética, países como Polonia, la República Checa, Eslovaquia y Hungría se volcaron sobre sí mismos en procesos de construcción institucional de democracias liberales así de transformación de sus economías hacia el libre mercado y la integración europea.

Pero también es cierto que hay fechas y momentos claramente definidos para entender los grandes cambios económicos, políticos y geopolíticos que dan lugar al concepto vigente -si bien dinámico- de Europa Central.

El primer momento es, en orden cronológico, la creación del llamado Grupo Visegrado en 1991 -integrado por Checoslovaquia, Hungría y Polonia y más tarde por Eslovaquia- que se asumió como portavoz de los intereses específicos y de las transformaciones de Europa Central.

El segundo momento fue el ingreso de esas naciones a la Organización del Tratado del Atlántico Norte - OTAN: la República Checa, Hungría y Polonia en 1999 y Eslovaquia en el 2004, que dio un giro de ciento ochenta grados a la ubicación geopolítica de esas naciones y a las nociones de seguridad europea.

El tercer momento fue el ingreso a la Unión Europea de esas cuatro naciones en el 2004, que impulsó cambios de gran velocidad hacia niveles insospechados de crecimiento y prosperidad y con una voz diferenciada en el concierto europeo sobre múltiples temas. 
Desde la perspectiva de diversas naciones, no parece haber gran claridad sobre las transformaciones de esta región que "tiene ahora un potencial de crecimiento mucho más rápido que el resto de la Unión Europea" (Dietl, M. 2021). Incluso, cataloga la próxima década (2021-2030) como el decenio de Europa Central con "un alto nivel de educación, un bajo desempleo y con un acceso al mercado interior (europeo) que reduce su vulnerabilidad a las crisis y hace de esta región un espacio atractivo para la inversión extranjera directa" (Bass, T. 2021).

Este trabajo se focaliza en una de las dimensiones más notables en la construcción de un nuevo concepto de Europa Central: la creación del Grupo Visegrado. A treinta años de distancia, nos pareció fundamental contar con una versión de primera mano de uno de los actores directos en la creación de ese grupo, el premio Nobel polaco Lech Walesa.

Vaclav Havel, József Antall, Walesa eran los presidentes de sus naciones cuando se creó el grupo Visegrado. Se trata de personajes que "revivieron la alguna vez desairada idea de Europa central, también ridiculizada e ignorada por muchos a lo largo de la primera mitad del siglo XX" (Daniška, J. 2018).

\section{2.- FUNDAMENTO TEÓRICO}

\section{El personaje}

Lech Walesa (n. 29 de octubre de 1943) es uno de los personajes míticos del siglo XX. Líder obrero del gran puerto industrial de Gdansk, al norte de Polonia, pionero del fin del comunismo y de la caída de la cortina de hierro en Europa del Este, mediante un diálogo pacífico y un proceso electoral. Premio Nobel de la Paz, presidente y estadista polaco. No siempre comprendido. Cuestionado y criticado hoy, especialmente en su propio país.

Relativamente popular en México, aunque en la década de los 1980s era muy difícil comprender que un proletario luchara por terminar con la dictadura del proletariado y del socialismo realmente existente, y que un líder obrero fuera católico y de centro-derecha.

Hoy, no cabe duda sobre su rol decisivo en ese momento histórico y, aún para cuestionarlo, Walesa siempre está en la mente de la población de Polonia y de aquellos que hablan sobre Polonia en cualquier parte del mundo. 
Lech Walesa se ubica en la categoría de mito con caracterizaciones diversas. Una, entrañable, es la película Walesa: Czlowiek z nadziei (Walesa: El hombre de la esperanza), del aclamado director Andrzej Wajda. Es una caracterización de coyuntura, a partir de una entrevista que la periodista italiana Oriana Fallaci hizo a Walesa en 1981 y que ella misma publicó bajo elocuente título, que no dio lugar a dudas: Walesa, el hombre que volvió loco al Kremlin.

La película de Wajda retrata al personaje, un obrero electricista, en su modesto y minúsculo departamento, con su familia -su esposa y seis niños que corren por aquí y por allá- pero, sobre todo, es un documento indispensable para entender la Polonia de principios de los 1980s y también el inicio del proceso que condujo al fin del comunismo en ese país, primero, y luego en toda Europa del Este.

El periodista estadounidense Douglas Stanglin, lo definió en un artículo de 1980 para United Press International como una persona de lenguaje "tosco" (salty-tongued), "duro, pero con sentido del humor", y un rasgo inconfundible: "su bigote de morsa y ropa arrugada (que) se han convertido en una marca internacional".

Pero, entre tantas, hay una caracterización ineludible de Walesa: la que hizo en su memorable Crónica de la Libertad, de 1983, el Nobel mexicano Octavio Paz:

"Su figura es emblemática de las fuerzas que mueven al pueblo polaco, -escribe Paz. Lech Walesa nació en un pueblo cercano a Varsovia. Su madre era campesina y su padre carpintero. Es electricista. Posee dos trajes y cinco pares de calcetines: un verdadero proletario. También un verdadero polaco tradicional: católico, nacionalista, generoso y sin mucho sentido del orden. Su mujer se llama...(Danuta): como llamarse Lupita en México o Carmen en España. La pareja tiene seis hijos. En 1976 Walesa fue despedido de los astilleros, estuvo varios meses sin empleo y vigilado por la policía. Fue uno de los organizadores de los sindicatos libres del Báltico. Walesa no es un intelectual ni un teórico. Tiene pocas ideas, mucho sentido común y un antiguo e instintivo sentido de la justicia. Es un hombre salido del pueblo y en su persona se funden dos tradiciones: la inmemorial de los campesinos con su cultura de siglos y la del proletariado industrial moderno" (Paz, Octavio. 1983). 


\section{El político}

El viaje de Walesa está marcado por los rasgos míticos del ascenso y la caída, el éxito y el fracaso. Y es en la política en donde se revela este destino.

El año de 1989 fue un año clave para Polonia y para la caída del primer ladrillo de la llamada cortina de hierro. Como parte de los de intensos eventos políticos en Polonia ese año, el sindicato Solidaridad no sólo fue legalizado de nuevo, luego de 7 años de clandestinidad (Si bien el sindicato Solidaridad se formó en septiembre de 1980 y fue reconocido como "sindicato legal" por las autoridades de la Polonia Popular, fue disuelto por los líderes comunistas en octubre de 1982, lo que obligó al movimiento a operar en la clandestinidad. Es hasta 1989 durante los llamados diálogos de la mesa redonda que el sindicato fue reconocido legalmente de nuevo), sino que alcanzó su cénit político, cuando Walesa lo transformó en la plataforma civil y política que abriría la puerta a la primera transición pacífica del comunismo a la democracia.

Se trató del Komitet Obywatelski "Solidarność (Comité Ciudadano del Presidente del Sindicato Solidaridad), que promovió candidatos a elecciones parlamentarias de ese año, luego de un ejercicio único conocido como la Mesa Redonda: un diálogo entre el gobierno y el movimiento Solidarność.

Esas elecciones parlamentarias dieron lugar al primer gobierno no comunista en Polonia desde el fin de la II Guerra Mundial, con lo cual se constituyó también como el primer país de la cortina de hierro que transitó pacíficamente a la democracia.

En esas elecciones parlamentarias de 1989 se disputaron ambas cámaras. Para la Cámara de Diputados o Sejm (de 460 miembros) el oficialista POUP (Partido Obrero Unificado de Polonia, PZPR por sus siglas en polaco) obtuvo 173 escaños seguido muy de cerca por "Solidarnośćc" que obtuvo 161; el resto de la oposición se repartió los 126 asientos restantes, con el cual el oficialismo comunista perdió la mayoría. Mientras tanto, en el Senado, el resultado fue apabullante: de 100 escaños en juego, el movimiento de Walesa obtuvo 99 asientos y el POUP sólo 1. Aún se recuerda una imagen: los candidatos de "Solidarność" hicieron campaña portando -todos, sin excepción- su fotografía acompañados de Lech Walesa.

Esas elecciones parlamentarias, en que triunfó la oposición democrática, fueron el prólogo a las elecciones presidenciales de 1990 en las que Walesa -de 47 años- obtuvo un apabullante triunfo con una 
votación con $74.25 \%$ de la votación en la segunda vuelta. Stephen Engelberg reportaría para The New York Times: "La escena frente a la sede de Solidaridad fue de descarada celebración. Miles de residentes de Gdansk vinieron a animar a su hijo más famoso y escuchar una banda de música. Detonaron petardos, arrojaron vasos contra la pared del cuartel general y abrieron botellas de champaña” (Engelberg, S. 1990).

Ese fue la cúspide política de Walesa y también las únicas elecciones presidenciales en que las obtuvo la victoria. Luego de su primer gobierno (1990-1995), buscó reelegirse sin éxito en 1995, con una votación, todavía apreciable, de $48.3 \%$ del total en la segunda vuelta.

Cinco años más tarde, en el 2000, se presentó por tercera vez como candidato a presidente, bajo el cobijo de un partido creado ad hoc: la Democracia cristiana de la 3ra República de Polonia. El resultado no sólo fue decepcionante, sino que prácticamente borró a Walesa del mapa político de Polonia.

Esas elecciones presidenciales estuvieron marcadas por dos rasgos. Primero, son, hasta hoy, las únicas elecciones presidenciales de la era democrática poscomunista en Polonia que no requirieron segunda vuelta, ya que hubo un candidato que, desde la primera vuelta, obtuvo más del $50 \%$ en elecciones de nutrida participación (más del 65\% del padrón electoral): Aleksander Kwaniewski, de la Alianza Izquierda Democrática, alcanzó el $54 \%$ de la votación. El segundo rasgo de esas elecciones fue, precisamente, la caída al precipicio político de Lech Walesa que obtuvo solamente el 1\% de la votación.

En el 2004 (Werkhäuser, N. 2004), Nina Werkhäuser para Deutsche Welle preguntó a Walesa:

- Muchos de los trabajadores que alguna vez lo apoyaron ya no votaron por usted. ¿Por qué?

\section{Walesa contestó:}

- El precio que pagamos por un cambio de sistema fue alto y ahora vemos los efectos en la alta tasa de desempleo, entre otras cosas. Pero deberíamos preguntar a los trabajadores: ¿De verdad quieren volver al comunismo? Soy la persona responsable de la situación actual, encabecé la batalla para llegar hasta aquí. Yo fui quien hizo esto, no Gorbachov. Por tanto, debo pagar el precio. Tengo una oficina, no gano tanto, pero mucha gente está desempleada y me tiene envidia. ¿Pero eso significa que me equivoqué al hacer lo que hice? Cuanta más gente se queje de mí hoy, más monumentos tendré mañana. 
Al parecer, con un dejo de amargura, años después Walesa comentó:

- Alguien dijo una vez que los vencedores nunca son juzgados, pero en este país sí lo son (Borger, J. 2011).

\section{La reunión con Walesa y su objetivo}

Decidimos acercarnos personalmente a Lech Walesa a propósito de este número de Política, Globalidad y Ciudadanía con un propósito específico: recibir de primera mano su visión de la construcción poscomunista de un nuevo concepto de la región de Europa Central, que contribuya a explicar por qué es esa, en la actualidad, una región de futuro con especificidades y características que significan un enorme potencial para la diversificación de las relaciones de México con países de la Unión Europea.

Walesa fue, junto con el presidente de la entonces Checoslovaquia, Vaclav Havel, protagonista central en la creación de un mecanismo creado exactamente hace 30 años, que se ha asumido como portador de los intereses específicos y de la identidad de Europa Central. Nos referimos al llamado Grupo Visegrado, que se formó en 1991, integrado originalmente por Checoslovaquia, Polonia y Hungría; la República Eslovaca se mantuvo también como parte de este grupo informal cuando se separó de la República Checa en 1993. Precisamente a partir de 1993 ese grupo es conocido simplemente como el V4.

Hoy día, se acepta que el Grupo Visegrado o V4 es la expresión política y de cooperación más consistente y clara de Europa Central. Los objetivos y grandes logros de este grupo en el primer periodo de su existencia se asociaron a la construcción de regímenes de democracia parlamentaria y economía de mercado, así como a su ingreso a la Unión Europea y a la OTAN luego de que, en efecto la República Checa, Hungría y Polonia ingresaran a la OTAN en 1999 -Eslovaquia lo haría en 2004- y que estas cuatro naciones accedieran a la Unión Europea en la ampliación de ese bloque en 2004.

El V4 se ha mantenido, hasta ahora, con un esquema triple: como grupo de presión política al interior de la Unión Europea; como grupo que ha desplegado un esfuerzo de integración y cooperación subregional; y como grupo que desarrolla, de una manera no necesariamente ordenada, vinculaciones con terceros actores y con otras regiones. 
En algunos círculos académicos y de opinión, parecería haber una visión probablemente estática y desactualizada en torno a esos países que son hoy, en realidad, naciones muy prósperas, educadas, de crecimiento económico constante y desarrollos impresionantes de infraestructura. El V4 en su conjunto tiene 65 millones de habitantes y sería la quinta economía más grande de Europa y la duodécima a nivel mundial. Cuenta también con 108 eurodiputados, es decir, poco más del 15\% del parlamento europeo. Aún más, en un contexto más amplio, no resulta claro que la política exterior mexicana haya logrado articular un enfoque de conjunto hacia los países que alguna vez formaron parte de la esfera soviética.

Es cierto que el concepto de Europa Central no es necesariamente claro. Hace tres décadas, por ejemplo, se debatía la existencia misma de Europa Central: "Durante casi cuarenta años después de 1945, el término estuvo casi completamente ausente del lenguaje político de Europa" (Timothy Garton Ash. 1999), explicaba Timothy Garton Ash en 1989, al perfilarse el fin del comunismo. Decía también que durante la Guerra Fría el continente europeo estuvo dividido simplemente entre Europa Occidental y Europa del Este (u Oriental). Agregaba: "En la década de 1980 (el concepto de Europa Central) fue revivido por escritores checos, húngaros y polacos como Milan Kundera, György Konrad y Czeslaw Milosz, como una alternativa intelectual y política a la Europa del Este dominada por los soviéticos", y también como un terremoto a las formas de pensamiento occidental ancladas en los conceptos obsoletos de la Guerra Fría. Milan Kundera también definía a Europa Central, en la misma época, como un Occidente secuestrado, una región con valores y fundamentos comunes, que habría sido secuestrada bajo la dominación soviética en la era comunista (Milan, Kundera. 1984).

También es cierto que hay intentos recientes de entender a Europa Central desde otros puntos de vista: "por un lado como el área entre los mares Báltico, Adriático y Negro (aunque esto sea demasiado simplista)" (Duda, A. 2021); por otro lado, como un espacio que busca nuevas formas organizativas de influencia en el contexto europeo -además de Visegrado-, como lo ejemplificarían el llamado "Nueve de Bucarest" (Formada en 2015, esta iniciativa polaco-rumana se inició como un foro para intercambiar opiniones y coordinar posiciones como "una voz fuerte y unida" sobre la seguridad de los países del flanco oriental de la OTAN: Polonia, Rumanía, Lituania, Letonia, Estonia, Hungría, Eslovaquia, República Checa y Bulgaria), y la Iniciativa croata-polaca Tres Mares (Trimarium) la cual es nacida en 2016, se trata de una iniciativa de cooperación entre los países situados alrededor del Mar Adriático, Mar Báltico y Mar Negro: Austria, Bulgaria, Croacia, República Checa, Estonia, Hungría, Letonia, Lituania, Polonia, Rumania, Eslovaquia y Eslovenia. La Iniciativa Tres Mares tiene como objetivo, sobre todo, 
fortalecer la integración económica de la región, así como el desarrollo de infraestructura de transporte, de energía y conectividad digital norte-sur.

No obstante, para efectos instrumentales y de diseño de políticas, la versión más consolidada de la subregión europea de Europa Central, al día de hoy, sigue estando constituida, como se ha dicho, por los países del llamado Grupo Visegrado -es decir, Eslovaquia, Hungría, República Checa y Polonia. El V4 se asume siempre en sus documentos como el grupo de "los países de región centroeuropea". Además de esfuerzos comunes al interior de la Unión Europea y de la búsqueda de proyectos comunes de cooperación, el V4 constituye también una de las regiones más prometedoras en términos de crecimiento económico, estabilidad y oportunidades para países como México.

En efecto, en la Declaración de los Primeros Ministros de la República Checa, Hungría, Polonia y la República Eslovaca con motivo del 30 aniversario del Grupo Visegrado (Cracovia, 17 de febrero de 2021), los Jefes de Gobierno observaron que “el Grupo Visegrado ha sido reconocido en Europa y a nivel mundial como un socio confiable y símbolo de una transformación política y económica exitosa, un pilar importante del proyecto histórico de la reunificación de Europa, así como un ejemplo de cooperación regional eficaz dentro de la Unión Europea, contribuyendo a su desarrollo, incluyendo, en ámbitos concretos, y para la competitividad económica del continente" (Declaration of the Prime Ministers of the Czech Republic. 2021).

\section{Escenario del encuentro con Walesa}

Octavio Paz dice también que "el tema central de la historia moderna de Polonia ha sido la pasión nacional y las tradiciones culturales y religiosas del pueblo polaco" (Paz, Octavio). Para quienes conocen Polonia, resulta claro que ese tema no es una abstracción del discurso político sino una experiencia que se revela cotidianamente en todas partes y en la mayor parte de los encuentros con mujeres y hombres de esa nación.

Nos encontramos con Walesa el 19 de agosto de 2020 en sus oficinas de en la ciudad portuaria de Gdansk. Con Walesa, la atmósfera deja siempre en claro que su mitología está construida de símbolos. Uno de ellos es, por supuesto, el Premio Nobel de la Paz, que su esposa Danuta recibió en su representación en 1983 y a cuya entrega no acudió Walesa por temor a que, a su regreso, no se le permitiera entrar de nuevo a su país. 
Negrín., A. \& Lozano., P.

Una crónica del diario español El País (José, Comas. 1983), publicada el 11 de diciembre de 1983, un día después de la entrega del Nobel, describió:

“El líder sindical polaco y premio Nobel de la Paz, Lech Walesa, siguió la ceremonia en Oslo de la entrega del galardón a su esposa, Danuta, a través de la retransmisión de la emisora norteamericana Radio Europa Libre, en la casa parroquial de la iglesia de Santa Brígida, situada en la zona del astillero Lenin, en Gdansk. Poco antes, en su casa, ante una multitud de periodistas, recordó la existencia de presos políticos que esperan proceso y advirtió contra los riesgos de manifestaciones callejeras.

"Walesa leyó en su domicilio un mensaje: En las cárceles hay muchos presos políticos, entre ellos 11 activistas y asesores de Solidaridad que esperan proceso. Desde hace dos años son rehenes por todos nosotros. Que todos los que me escuchan repitan detrás de mí: 'Si me llego a olvidar de ellos, Tú, Dios, olvídate de mí'. Esto es un juramento y todas las entrevistas conmigo se encabezarán así. Mientras hablaba con los periodistas, bajo una pintura del papa polaco Juan Pablo II, Walesa firmaba autógrafos sobre una estampa que decía: Acto de entrega a la Madre de Dios de Lech Walesa, con un dibujo del águila polaca con la Virgen dentro de su cuerpo. Al dorso, la estampa reproduce las palabras de Walesa al encomendar a la Virgen Negra de Jasna Gora (Czestochowa), el 21 de octubre de 1980, el sindicato Solidaridad".

El segundo símbolo es, precisamente, el lugar en que nos encontramos esa soleada tarde del verano del 2020: el Centro Europeo de Solidaridad (CES), creado por Walesa para asegurar la vigencia y continuidad de sus valores. Se trata de una impresionante construcción ubicada en la plaza Solidarności, justo a la entrada del famoso astillero de la ciudad báltica de Gdansk (Astillero Lenin en la era comunista), y donde tuvo lugar la histórica huelga de agosto de 1980 que dio lugar al nacimiento del sindicato Solidaridad, en septiembre de ese año. El edificio, de 30 metros de altura, es una extraordinaria representación arquitectónica de una enorme embarcación: sus paredes exteriores están revestidas con láminas de acero de color óxido, que provocan una ilusión óptica marina, en un conjunto complementado por el Monumento a los trabajadores de los astilleros navales caídos en las protestas contra el régimen comunista en 1970.

140 Revista Política, Globalidad y Ciudadanía | Vol. 8, Núm. 15, enero - junio 2022 | ISSN 2395-8448 | http://revpoliticas.uanl.mx/ 
La oficina de Walesa se encuentra en el piso II del CES. Al entrar, se respira en cada rincón el personal universo iconográfico de Walesa que encierra toda una cultura: un gran crucifijo y un cuadro que retrata al Papa polaco, Juan Pablo II (Karol Wojtyla). En un guiño peculiar, la oficina de Walesa se encuentra adornada también con una versión contemporánea de La última cena de Leonardo da Vinci, que, al mismo tiempo, representa los históricos Acuerdos de la Mesa Redonda (negociados entre el 6 de febrero y el 4 de abril de 1989), que, como se dijo anteriormente, dieron lugar a unas elecciones parlamentarias en Polonia que, a su vez, marcaron el inicio del fin de la cortina de hierro en Europa. En la pintura, el rostro de Walesa se sobrepone al de Jesús y los rostros de los doce apóstoles son los de aquellos quienes lo acompañaron en la negociación de esos acuerdos, políticos e intelectuales que han marcado la historia más reciente de Polonia: Jacek Kuroń, Andrzej Stelmachowski, Tadeusz Mazowiecki, Jarosław Kaczyński, Andrzej Wielowieyski, Bronisław Geremek, Władysław Frasyniuk, Adam Michnik, Jerzy Turowicz, Arkadiusz Bratkowski, Jan Józef Szczepański y Aleksander Hall.

Después de que nos fuera medida la temperatura como disposición preventiva en medio de la pandemia del covid-19, y todos con cubre bocas, Walesa nos recibió de buen humor.

Vestía una playera blanca con la palabra “Grundgesetz” (Constitución, en alemán) en letras rojas. Si en 1980 se le veía desaliñado, en 2020, 40 años más tarde, no parece diferente.

A sus 77 años, viste usualmente playeras con la palabra Constitución para revindicar el valor de la legalidad y protestar, según él, contra "violaciones" a las leyes polacas. Por ejemplo, en 2018, en el funeral de Estado del presidente George H.W. Bush en Washington, asistió con una camiseta gris -bajo un tweed negro- con la misma leyenda "Konstytucja". Walesa declaró en esa ocasión que "era su gesto personal de despedida a un líder estadounidense que luchó por la libertad, luchó por la verdad y la honestidad" (AP, Vanessa Gera. 2018).

Walesa es ya un viejo lobo de las entrevistas y las conferencias y rompe el hielo con facilidad. Nos dice que ha estado al menos 10 veces en México y que, por cierto, Krzysztof Smolana en su libro "Polonia y México a lo largo de la historia: una perspectiva de la misión diplomática polaca" (SRE, Instituto Matías Romero, 2018) recuerda, por ejemplo, la visita de Walesa - "muy estimado en México"en 1996, en la que recibió un doctorado honoris causa por la Universidad Anáhuac. Smolana anota que Walesa "visitó posteriormente México en varias ocasiones en viajes no oficiales". Recuerda la última de esas visitas, en septiembre del 2019, con motivo de la 17 Cumbre Mundial de los Premios Nobel de la 
Paz, en Mérida, Yucatán. Nos dice con enorme deferencia: "si mi primera visita a México la hubiera hecho de joven, probablemente me habría quedado allí. No habría participado en Solidarność porque habría estado en México".

Se refiere también a la coyuntura específica de la relación entre México y los Estados Unidos, y en específico al famoso muro promovido por el entonces presidente Donald Trump. Nos dice que las nuevas tecnologías facilitan que las sociedades se conozcan y, tajante, comenta: "cerrar fronteras no es la solución”. Recuerda que en un encuentro que tuvo con Donald Trump (probablemente este encuentro tuvo lugar en la visita de Trump a Varsovia los días 5 y 6 de julio de 2017), le habría dicho que "no creía en sus muros" y había criticado su iniciativa del muro con México: "Creo que por eso le caigo mal a Trump", anotó.

La opinión de Walesa sobre Trump fue cambiando con el tiempo durante la presidencia de este último. En 2016, tras la elección en la que resultara electo Donald Trump, Walesa publicaría en su cuenta de Facebook una foto de una reunión entre ambos en 2010 en Mar-a-Lago en Palm Beach, Florida.

En esa ocasión Walesa diría que "Si en Polonia fue posible que un trabajador derrocara el comunismo y se convirtiera en presidente, ¿por qué un millonario no puede convertirse en presidente en la América capitalista ...? Como pueden ver, mi historia fue una inspiración para que actuara”. En 2018, criticaba la construcción del muro ("La pregunta es qué vas a construir. Para qué estás rompiendo si no sabes cómo construir y es aquí en donde no estoy de acuerdo con él") y criticaba ya las corrientes populistas. En 2019, y con motivo de las celebraciones del 80 aniversario del comienzo de la 2da Guerra Mundial, diría que "Estados Unidos ya no era la principal potencia mundial” y urgía a Trump a recuperar “el liderazgo moral y político".

\section{Los padres fundadores de la nueva Europa Central}

Felicitamos a Walesa porque muy recientemente se había anunciado que el Ministerio de Cultura de Polonia buscaría que los astilleros de Gdansk fueran inscritos en la lista de Patrimonio Mundial de la UNESCO por ser un "símbolo de la defensa de los ideales de libertad, simbolizado, sin importar el tiempo, por la idea de solidaridad, que unió a millones de habitantes del antiguo Bloque Oriental en la lucha contra el régimen comunista" (UNESCO). 
Recordamos también su muy famosa entrevista con Oriana Fallaci en 1981 en la que dijo: "No soy diplomático, no soy maestro de ceremonias y mucho menos intelectual. Soy un hombre tosco, trabajador". Nos dice ahora con una sonrisa: “Oriana me cayó de sorpresa... prácticamente me obligó a hacer la entrevista".

Entramos directamente a nuestro tema recordando que un 15 de febrero de 1991 se creó el llamado Grupo Visegrado en una cumbre de jefes de Estado y de gobierno de la entonces Checoslovaquia, Hungría y Polonia. Anotamos que, en 2021, precisamente, se celebran los 30 años del nacimiento del V4 y que es ineludible recordar a sus padres fundadores, los entonces mandatarios de esas naciones: Václav Havel, Joszef Antall y el propio Lech Walesa, quienes revolucionaron la microhistoria de esta región centroeuropea.

Walesa se retrotrae al contexto de la transformación de Polonia, a la caída de la Polonia Popular y al nacimiento de lo que define como una nueva república. "Se trata de una iniciativa que fue en parte fruto de la idea de Solidaridad, lanzada años antes que Visegrado”.

Reflexiona que su participación en la creación del V4, como presidente de Polonia, se dio en periodo no sólo de transformación sino de construcción de todo un nuevo sistema político, económico, y de construcción democrática. Agrega que con Visegrado "había que romper las barreras... había que cambiar el mundo y enfrentar el monopolio comunista", en especial a través de la integración regional lo cual ha sido uno de los logros más significativos del V4. Sin embargo, considera que ahora se requiere "una nueva era, una segunda ola de nuevas soluciones, hacer todo un nuevo sistema".

Recordamos que hace exactamente tres lustros, con motivo del 15 aniversario del V4, Walesa escribió: "Mirando hacia atrás... podemos confirmar que el Grupo de Visegrado se fundó sobre objetivos mutuos de política exterior, experiencias históricas similares y proximidad geográfica. Estos terrenos comunes todavía proporcionan un punto de referencia sustancial en nuestras relaciones mutuas" (Walęsa, Lech).

Comentamos también que el éxito del V4 es resultado de la construcción de una nueva identidad centroeuropea por lo menos con tres elementos: profundas raíces históricas; la integración a la Unión Europea y la democratización y establecimiento del libre mercado en esas naciones, luego del fin de la era comunista. 
En su ensayo "Un milagro llamado Visegrado", Martin Bútora dice que "como concepto político, la identidad centroeuropea estaba ligada a un terreno problemático, que había sido testigo de dramas que más de una vez llevaron a un conflicto global" y que no solamente estaba relacionado con "las personalidades y los estadistas que habían liderado la lucha por la libertad, desde Kościuszko hasta Kossuth y Masaryk y los luchadores modernos por la democracia como Lech Walesa, Václav Havel y Arpád Göncz" (Publicado en el libro "The Visegrad Group: A Central European Constellation", ed. por Andrzej Jagodziński, International Visegrad Fund, Bratislava, 2006).

\section{El regreso a Europa}

Comentamos a Walesa que, sin duda, otro de los padres fundadores y signatarios del acta fundacional del V4 en 1991 fue Václav Havel (1936-2011), último presidente de Checoslovaquia y primer presidente de la República Checa.

Una de las razones por las que recordamos a Havel es por su notable contraste con Walesa. El escritor y analista político checo Jiří Pehe escribió: "Una sucesión de formas autoritarias de gobierno y regímenes semidemocráticos obligó a los intelectuales de la región a fungir como sustitutos de los políticos en el discurso político. Los intelectuales y figuras culturales en particular jugaron un papel político, directa o indirectamente, que va mucho más allá de lo común en las democracias establecidas" (Pehe, J.). Sin embargo, la misma coyuntura daría lugar a la emergencia de un líder obrero, un electricista de humilde origen como Walesa.

Otra razón para evocar a Havel es su coincidencia de objetivos con Walesa. De visita en Polonia, el 25 de febrero de 1990, Havel pronunció un histórico discurso en el Sejm (cámara baja del parlamento de Polonia). Ahí anunció la formación -que se concretaría un año más tarde- del Grupo Visegrado. Havel dijo en esa ocasión:

"Los años de destinos y luchas similares por ideales similares deben evaluarse a la luz de la amistad genuina y del respeto mutuo; es decir, precisamente el espíritu que dominó aquellos años durante los cuales la literatura secreta independiente era contrabandeada en mochilas a través de nuestras cordilleras comunes (...) Esa amistad auténtica, basada en una conciencia similar del destino impuesto a nuestros dos países, en las lecciones comunes que aprendimos, y sobre todo en los ideales comunes que ahora nos unen, deberían conducir en última instancia a una 
coordinación adecuada de nuestras políticas, en un proceso al que ambos nos referimos y entendemos como el regreso a Europa. También deberíamos coordinar nuestros esfuerzos lo mejor que podamos con Hungría... y con otras naciones de esta región de Europa. No deberíamos competir entre nosotros para ser admitidos en las distintas organizaciones europeas. Al contrario, debemos ayudarnos unos a otros con el mismo espíritu de solidaridad con el que, en días más oscuros, protestaron por nuestra persecución como nosotros lo hicimos contra la suya.

Es demasiado pronto para predecir qué formas institucionales adoptará nuestra coordinación en Europa central y oriental. Europa occidental está considerablemente adelante de nosotros en sus procesos de integración, y, si cada uno de nuestros países regresara a Europa por separado, podría llevar mucho más tiempo y sería un proceso mucho más complejo que si procediéramos de manera coordinada. Esa (integración) concierne no sólo a la economía: concierne a todo, incluidas las conversaciones sobre desarme.

Muy pronto, me gustaría invitar a varios representantes del Estado y del pueblo de Polonia y Hungría, quizás con observadores de otros países de Europa Central, a una reunión en el Castillo de Bratislava -hoy capital de Eslovaquia- donde podríamos pasar un día hablando tranquilamente sobre estos asuntos. Quizás esto nos haría un poco más sabios.

De cualquier forma, una cosa es segura: por primera vez en la historia, tenemos una oportunidad real de llenar con algo realmente significativo el gran vacío político que quedó en Europa Central después del colapso del Imperio de los Habsburgos. Tenemos la oportunidad de transformar Europa Central de lo que fue un fenómeno principalmente histórico y espiritual a un fenómeno político. Tenemos la oportunidad de tomar esta guirnalda de estados europeos -hasta hace poco colonizados por la Unión Soviética y que ahora intentan construir una relación con ese país basada en la igualdad- y convertirla en un cuerpo especial. Entonces podremos acercarnos a las naciones más ricas de Europa occidental, no como pobres fracasados o prisioneros indefensos, recientemente amnistiados, sino como países que pueden hacer una contribución real. Lo que tenemos para ofrecer son impulsos espirituales y morales, iniciativas de paz valientes, un gran potencial creativo poco explotado y un espíritu especial creado por nuestra libertad recién ganada. Podemos ofrecer la inspiración para considerar soluciones rápidas y atrevidas" (The Visegrad Group: the Czech Republic, Hungary, Poland and Slovakia). 
El planteamiento de Havel refiere a la resurrección de un conjunto de naciones luego de la era comunista, no sólo como uno de los grandes hechos de la historia europea contemporánea, sino como un tema identitario, que persiste hasta hoy, incluso en el contexto integracionista de la Unión Europea.

Se ha dicho que la creación del V4 debe entenderse como la formación de una región "que tuvo que luchar la mayor parte del tiempo por su propia identidad, apretujada entre Oriente y Occidente" (The Visegrad Group: the Czech Republic, Hungary, Poland and Slovakia). Aún más, que los países de Visegrado se ven a sí mismos como la verdadera Europa: una que aprecia enormemente valores como el estado nacional, la familia, y el cristianismo occidental frente al cristianismo ortodoxo, y los vive como las expresiones ecos más auténticas de la tradición europea. Por ello, incluso diferencias que algunos países del V4 han temido en tiempos recientes con las instituciones europeas de Bruselas no obedecerían en modo alguno -según esta interpretación- a un sentimiento antieuropeo sino a una batalla sobre lo que significa ser europeo.

Al recordar a Vaclav Havel, Walesa sonríe. Nos comenta que "nos hicimos muy buenos amigos". Medita un momento y comenta: "las ideas de Havel eran teóricas, muy interesantes. Havel era muy inteligente, pero sus ideas no eran necesariamente prácticas. Nos complementábamos muy bien: yo soy muy práctico, yo llego a la teoría a través de la práctica y no al revés. Si Havel y yo pudiéramos trabajar juntos ahora, podríamos hacer muchas cosas... podríamos llegar a la cabeza de los teóricos”.

En el 2011, tras el fallecimiento de Havel el 18 de diciembre, la Agencia de Prensa Polaca (PAP) recoge una entrevista de Walesa con el diario italiano La Repubblica y en la que comentaría algo similar: "Él era un hombre valiente con la pluma mientras yo dirigía a los trabajadores. Havel era un teórico perfecto, aunque no un gran organizador. Luchó con las palabras y la pluma y lo pagó personalmente. Era un hombre de palabras y sus palabras empujaron a otros a organizarse" (Wałęsa: gdyby nie Havel, Polacy zostaliby sami).

Walesa declararía en dicha ocasión también que, si no hubiera sido por la llamada Revolución de Terciopelo en la entonces Checoslovaquia, liderada por Havel, los polacos se habrían quedado solos: "es cierto que los checos nunca hubieran ganado si nosotros no hubiéramos ganado antes en 1989, pero hay algo más que decir. Si la Revolución de Terciopelo en Checoslovaquia no hubiera ganado, y si el comunismo sólo se hubiera derrumbado en Polonia, nosotros, los polacos, nos hubiéramos quedado solos. Y luego nos hubieran pacificado" (Íbidem). 


\section{Comentarios sobre Generalidades}

El fin del comunismo fue un proceso aparejado por el surgimiento de liderazgos asociados a nuevas formas de construcción de la democracia. Walesa, Havel y Gorbachov figuran entre esos líderes. Por eso, la fundación creada por Walesa tiene como prioridad la educación sobre "las reglas de la democracia", que es también un tema recurrente en sus conferencia, reuniones y entrevistas.

La nuestra fue la excepción y Walesa resolvió decidió abordar, como último tema de nuestro encuentro, ese que tanto le apasiona. Comenta que hay que "reconocer que la democracia funciona bastante bien cuando hay división de poderes". Sin embargo, explica que "el simple hecho de votar significa democracia”. Destaca la importancia de la educación y del civismo y que hace falta una reorganización de la actividad de los ciudadanos ya que en muchos países son bajos los porcentajes de participación: ¿Si la democracia es mayoría, y, por ejemplo, solamente el 50\% vota, entonces: dónde está la democracia? Exagerando, pero para ilustrar su punto, dice: "llegaremos al momento en que solamente los candidatos voten".

Walesa reflexiona: “Cómo puede funcionar de verdad la democracia?” Nos habla de lo que él define como la Fórmula Walesa sobre la democracia, constituida por tres elementos: el primer elemento, "un primer 30\% de la fórmula", lo componen los derechos: la constitución, las leyes, las regulaciones. “El segundo $30 \%$ es la percepción de la gente sobre la democracia y cómo se comporta la gente en la democracia”, es decir si la gente participa en las elecciones, si la gente vota, si se encuentra organizada en partidos políticos o en otras estructuras. El "tercer 30\% es la chequera, el tamaño de la cuenta de banco de la gente". Saber si se cuenta con los recursos para “afrontar económicamente la democracia”. En cuanto a este último factor económico-social de la democracia, Walesa anota: "sólo un porcentaje muy bajo de la población se puede oponer a cambios antidemocráticos ya que (hay países en que un alto porcentaje de la población) no se puede permitir luchar u oponerse si no tiene el capital para comer, para sobrevivir, para hacer huelgas: la gente tiene miedo a perder su trabajo".

Hacemos notar que los tres ingredientes de la democracia que nos ha comentado suman un 90\%: “el 10\% faltante (de la fórmula) puede ser lo que ustedes quieran que sea”, concluye. 


\section{3.- MÉTODO}

El presente artículo es un caso de estudio contextualizado a partir de la entrevista semiestructurada de una fuente directa y primaria, para concluir con un texto explicativo construido a partir de un método sintético que analiza, detalla y sintetiza la información recopilada a fin de dar mayor contenido a la conversación que tuvieron los autores con el expresidente de Polonia, Lech Walesa. Finalmente, se detalla que se trata de una investigación cualitativa, ya que se exploraran las experiencias interpretativas -y hasta subjetivas- del entrevistado.

Este análisis considera como participantes al expresidente de Polonia Lech Walesa, a México como parte interesada en el desarrollo de una política exterior hacia la región de estudio, así como a los países que conforman Europa Central, que para efectos de este estudio se considera así a los miembros del Grupo Visegrado: Polonia, República Checa, Eslovaquia y Hungría.

Para construir una Visión de Europa Central, se realizó una recopilación, y selección documental de fuentes primarias provenientes de distintos gobiernos de la región de Europa Central y especialmente la propia conversación de los autores con el expresidente de Polonia, Lech Walesa, considerando sus experiencias como fuente directa y primaria. En el caso de fuentes secundarias, y con el propósito de proveer contexto, se consideraron estudios académicos sobre la región centroeuropea; artículos y reportes periodísticos históricos; así como una selección de entrevistas realizadas previamente al expresidente de Polonia Lech Walesa con la finalidad de hacer un análisis comparativo y comprensivo de la conversación sostenida por los autores.

\section{4.- CONCLUSIONES}

Como se ha explicado en este trabajo, el concepto de Europa Central ha evolucionado a lo largo de la historia. Desde del fin del comunismo y hasta nuestros días, es una región que se resignificó, en especial a partir de la agrupación de cuatro naciones (Polonia, República Checa, Eslovaquia y Hungría) en el llamado Grupo Visegrado en 1991 y que se asumió como representante de Europa Central.

Las profundas transformaciones de esa región, luego de la caída de la cortina de hierro, han tenido diversos anclajes: el tránsito hacia economías de mercado y democracias liberales, y, por otro lado, su integración a la Unión Europea y su reposicionamiento estratégico desde su membresía a la OTAN. 
Los resultados de treinta años de cambios -tan radicales como exitosos- son notables y culminan en su salto a un nivel de desarrollo cercano a las economías más prósperas del continente europeo, patente en ámbitos como la calidad de la educación, el desarrollo de infraestructuras y la complejidad de su estructura productiva.

Desde el enfoque de naciones como México, entender el concepto actual de Europa Central tiene un sentido instrumental. Por un lado, porque se trata de una subregión que ofrece grandes oportunidades para México, a partir de sus especificidades, en el contexto de la Unión Europea. Por otro lado, porque México ha buscado articular un marco más eficaz hacia las naciones que conformaron el antiguo bloque comunista en Europa. Luego de un periodo en que las naciones del Grupo Visegrado estuvieron inmersas en profundas transformaciones como parte de su integración a la Unión Europea, han emergido como actores en internacionalización, buscando socios estratégicos más allá del continente europeo.

La entrevista que realizamos a Lech Walesa, uno de los artífices del concepto vigente de Europa Central y de la caída de la cortina de hierro, registra matices y profundiza en una visión actualizada, indispensable para el conocimiento de esa región.

\section{REFERENCIAS}

Bass, T. (2021, enero 22). 2021-2030, la décennie de l'Europe centrale. https://www.lopinion.fr/edition/international/2021-2030-decennie-l-europe-centrale-234040

Borger, J. (2011, April 04). Lech Walesa: The man who 'never made a Mistake' sees errors all around. (The Guardian). https://www.theguardian.com/world/2011/apr/04/lech-walesa-sees-errors-allaround-poland

Comas, J. (1983, 11 de diciembre). Walesa vivió desde Gdansk la entrega del Nobel de la Paz entre $\begin{array}{lllll}\text { periodistas } & y & \text { champaña }\end{array}$ https://elpais.com/diario/1983/12/11/internacional/439945207_850215.html

Daniška, J. (2018). Visegrad: From intellectual idea to political reality. International Issues \& Slovak Foreign Policy Affairs, 27(1-2), 43-53. Doi: 10.2307/26592068

Dietl, M. (2021, Enero 22). L'Europe centrale et son potentiel de forte croissance. https://www.lopinion.fr/edition/international/l-europe-centrale-potentiel-forte-croissancetribune-marek-dietl-234039 
Duda, A. (2021, enero 22). L'Europe centrale, comme une communauté des aspirations. https://www.lopinion.fr/edition/international/l-europe-centrale-communaute-aspirationstribune-d-andrzej-duda-234030

Engelberg, S. (1990, Diciembre 10). Poland elects WALESA president in landslide (The New York times). https://www.nytimes.com/1990/12/10/world/poland-elects-walesa-president-inlandslide.html

France 24 (2019). Walesa to Trump: US no longer moral, political world leader. https://www.france24.com/en/20190829-walesa-to-trump-us-no-longer-moral-political-worldleader

Garton Ash, T. (1999). The Puzzle of Central Europe. The New York Review of Books.

Gera, V. (2018). (diciembre 6). Poland's Lech Walesa wears protest T-shirt to Bush funeral (AP). https://apnews.com/article/51266ab4a3dc4433b0c25d1637cad0e8

Kaminski, A., \& Kurczewsha, J. (1991). Letter from Poland. Government and Opposition, 26(2), 215228. Retrieved March 16, 2021, from http://www.jstor.org/stable/44482574

Lee, C. \& Bideleux, Robert. (2012). East, West, and the Return of 'Central': Borders Drawn and Redrawn. 10.1093/oxfordhb/9780199560981.013.0004.

Milan, K. (1984). the Tragedy of Central Europe (Ed. 31. 7). The New York Review of Books.

Paz, O. (1983). Crónica de la libertad. Tiempo Nublado, Seix Barral.

Pehe, J. (2002). Central European identity in politics. http://www.pehe.cz/prednasky/2002/centraleuropean-identity-in-politics

RPP Noticias (2021). Lech Walesa criticó el muro fronterizo de Donald Trump: "La pregunta es qué vas a construir"

https://rpp.pe/peru/actualidad/donald-trump-nobel-de-la-paz-lech-walesa-critico-muro-fronterizo-lapregunta-es-que-vas-a-construir-noticia-1163351?ref=rpp

Simpson, P. (1996). The Troubled Reign of Lech Wałesa in Poland. Presidential Studies Quarterly, 26(2), 317-336. Retrieved March 16, 2021, from http://www.jstor.org/stable/27551580

Sinnhuber, K. A. (1954). Central Europe: Mitteleuropa: Europe Centrale: An Analysis of a Geographical Term. Transactions and Papers (Institute of British Geographers), 20, 15. https://doi.org/10.2307/621131

Stanglin, D. (1980). (noviembre 30). Personality Spotlight: Lech Walesa -- Polish labor leader: He challenged communist leaders and won. 
https://www.upi.com/Archives/1980/11/30/Personality-Spotlight-Lech-Walesa-Polish-laborleaderNEWLNHe-challenged-communist-leaders-and-won/1711344408400/

Terenzani, M. (2010). A common but contentious history (Visegrad Group). https://www.visegradgroup.eu/other-articles/common-but-contentious

UNESCO (2021). Gdansk - Town of Memory and Freedom. https://whc.unesco.org/en/tentativelists/530/ Visegrad Group (2021). Declaration of the Prime Ministers of the Czech Republic, Hungary, the Republic of Poland and the Slovak Republic on the Occasion of the 30th Anniversary of the Visegrad Group. https://www.visegradgroup.eu/calendar/2021/declaration-of-the-prime

Visegrad Group (2021). Havel, Václav: Speech in the Polish Parliament (Sejm). https://www.visegradgroup.eu/the-visegrad-book/havel-vaclav-speech-in

Visegrad Group (2021). Wałęsa, Lech: From Solidarność (Solidarity) to Cooperation and Integration. https://www.visegradgroup.eu/the-visegrad-book/walesa-lech-from

Werkhäuser, N. (2004). (Mayo 26). Interview with Lech Walesa (DW). https://www.dw.com/en/interview-with-lech-walesa/a-1217536

Wprost (2011). Watęsa: gdyby nie Havel, Polacy zostaliby sami. https://www.wprost.pl/przegladprasy/285120/walesa-gdyby-nie-havel-polacyzostalibysami.html?utm_source=wprost.pl\&utm_medium=recommendation\&utm_campaign=a utoload-return 